\title{
Archimedean Atomic Lattice Effect Algebras with Complete Lattice of Sharp Elements ${ }^{\star}$
}

\author{
Zdenka RIEČANOVÁ
}

Department of Mathematics, Faculty of Electrical Engineering and Information Technology, Slovak University of Technology, Ilkovičova 3, SK-812 19 Bratislava, Slovak Republic

E-mail: zdenka.riecanova@stuba.sk

Received September 29, 2009, in final form January 04, 2010; Published online January 06, 2010 doi:10.3842/SIGMA.2010.001

\begin{abstract}
We study Archimedean atomic lattice effect algebras whose set of sharp elements is a complete lattice. We show properties of centers, compatibility centers and central atoms of such lattice effect algebras. Moreover, we prove that if such effect algebra $E$ is separable and modular then there exists a faithful state on $E$. Further, if an atomic lattice effect algebra is densely embeddable into a complete lattice effect algebra $\widehat{E}$ and the compatiblity center of $E$ is not a Boolean algebra then there exists an $(o)$-continuous subadditive state on $E$.
\end{abstract}

Key words: effect algebra; state; sharp element; center; compatibility center

2010 Mathematics Subject Classification: 06C15; 03G12; 81P10

\section{Introduction, basic definitions and some known facts}

The classical (Kolmogorovian) probability theory is assuming that every two events are simultaniously measurable (as every two elements of a Boolean algebra are mutually compatible). Thus this theory cannot explain events occuring, e.g., in quantum physics, as well as in economy and many other areas.

Effect algebras generalize orthomodular lattices (including noncompatible pairs of elements) and $M V$-algebras (including unsharp elements, meaning that $x$ and non $x$ are not disjoint). Thus effect algebras may be carriers of probability measures when elements of these structures represent properties, questions or events with fuzziness, uncertainty or unsharpness, and which may be mutually non-compatible. For equivalent (in some sense) structures called D-posets introduced by F. Kôpka and F. Chovanec we refer the reader to [3] and citations given there.

Effect algebras were introduced by D.J. Foulis and M.K. Bennet (see [4]) for modelling unsharp measurements in a Hilbert space. In this case the set $E(H)$ of effects is the set of all self-adjoint operators $A$ on a Hilbert space $H$ between the null operator 0 and the identity operator 1 and endowed with the partial operation + defined iff $A+B$ is in $E(H)$, where + is the usual operator sum. The effect algebra $E(H)$ is called a standard effect algebra of operators on Hilbert space (or, a standard Hilbert space effect algebra, for short).

In approach to the mathematical foundations of physics the fundamental notions are states, observables and symmetries. D.J. Foulis [5] showed how effect algebras arise in physics and how they can be used to tie together the observables, states and symmetries employed in the study of physical systems. Moreover, that due to work of G. Ludwig [14] and subsequent book [2] by P. Bush, P.J. Lahti and P. Mittelstaedt a fourth fundamental notion, called effect, should be appended to this list.

\footnotetext{
${ }^{\star}$ This paper is a contribution to the Proceedings of the 5-th Microconference "Analytic and Algebraic Methods V". The full collection is available at http://www.emis.de/journals/SIGMA/Prague2009.html
} 
In spite of the fact that effect algebras are very natural algebraic structures to be carriers of states and probability measures, in above mentioned non-classical cases of sets of events, there are effect algebras admitting no states and hence also no probability measures (see [22]). Questions about the existence of states on effect algebras are at present time only partially solved. They are known only some families of effect algebras with states.

We study Archimedean atomic lattice effect algebras with a complete lattice of sharp elements as well as such which are densely embedable into complete atomic lattice effect algebras. For these effect algebras we give conditions under which states on them exist.

Definition 1 ([4]). A partial algebra $(E ; \oplus, 0,1)$ is called an effect algebra if 0,1 are two distinct elements and $\oplus$ is a partially defined binary operation on $E$ which satisfy the following conditions for any $x, y, z \in E$ :

(Ei) $\quad x \oplus y=y \oplus x$ if $x \oplus y$ is defined,

(Eii) $\quad(x \oplus y) \oplus z=x \oplus(y \oplus z)$ if one side is defined,

(Eiii) for every $x \in E$ there exists a unique $y \in E$ such that $x \oplus y=1$ (we put $x^{\prime}=y$ ),

(Eiv) if $1 \oplus x$ is defined then $x=0$.

We often denote the effect algebra $(E ; \oplus, 0,1)$ briefly by $E$. On every effect algebra $E$ the partial order $\leq$ and a partial binary operation $\ominus$ can be introduced as follows:

$x \leq y$ and $y \ominus x=z$ iff $x \oplus z$ is defined and $x \oplus z=y$.

If $E$ with the defined partial order is a lattice (a complete lattice) then $(E ; \oplus, 0,1)$ is called a lattice effect algebra (a complete lattice effect algebra).

Definition 2. Let $E$ be an effect algebra. Then $Q \subseteq E$ is called a sub-effect algebra of $E$ if

(i) $1 \in Q$,

(ii) if out of elements $x, y, z \in E$ with $x \oplus y=z$ two are in $Q$, then $x, y, z \in Q$.

If $E$ is a lattice effect algebra and $Q$ is a sub-lattice and a sub-effect algebra of $E$ then $Q$ is called a sub-lattice effect algebra of $E$.

Note that a sub-effect algebra $Q$ (sub-lattice effect algebra $Q$ ) of an effect algebra $E$ (of a lattice effect algebra $E$ ) with inherited operation $\oplus$ is an effect algebra (lattice effect algebra) in its own right.

For an element $x$ of an effect algebra $E$ we write $\operatorname{ord}(x)=\infty$ if $n x=x \oplus x \oplus \cdots \oplus x$ ( $n$-times) exists for every positive integer $n$ and we write $\operatorname{ord}(x)=n_{x}$ if $n_{x}$ is the greatest positive integer such that $n_{x} x$ exists in $E$. An effect algebra $E$ is Archimedean if $\operatorname{ord}(x)<\infty$ for all $x \in E$.

A minimal nonzero element of an effect algebra $E$ is called an atom and $E$ is called atomic if under every nonzero element of $E$ there is an atom.

For a poset $P$ and its subposet $Q \subseteq P$ we denote, for all $X \subseteq Q$, by $\bigvee_{Q} X$ the join of the subset $X$ in the poset $Q$ whenever it exists.

We say that a finite system $F=\left(x_{k}\right)_{k=1}^{n}$ of not necessarily different elements of an effect algebra $(E ; \oplus, 0,1)$ is orthogonal if $x_{1} \oplus x_{2} \oplus \cdots \oplus x_{n}$ (written $\bigoplus_{k=1}^{n} x_{k}$ or $\bigoplus F$ ) exists in $E$. Here we define $x_{1} \oplus x_{2} \oplus \cdots \oplus x_{n}=\left(x_{1} \oplus x_{2} \oplus \cdots \oplus x_{n-1}\right) \oplus x_{n}$ supposing that $\bigoplus_{k=1}^{n-1} x_{k}$ is defined and $\bigoplus_{k=1}^{n-1} x_{k} \leq x_{n}^{\prime}$. We also define $\bigoplus \varnothing=0$. An arbitrary system $G=\left(x_{\kappa}\right)_{\kappa \in H}$ of not necessarily different elements of $E$ is called orthogonal if $\bigoplus K$ exists for every finite $K \subseteq G$. We say that for an orthogonal system $G=\left(x_{\kappa}\right)_{\kappa \in H}$ the element $\bigoplus G$ exists iff $\bigvee\{\bigoplus K \mid K \subseteq G$ is finite $\}$ exists in $E$ and then we put $\bigoplus G=\bigvee\{\bigoplus K \mid K \subseteq G$ is finite $\}$. (Here we write $G_{1} \subseteq G$ iff there is $H_{1} \subseteq H$ such that $\left.G_{1}=\left(x_{\kappa}\right)_{\kappa \in H_{1}}\right)$. 


\section{The center of Archimedean atomic lattice effect algebra with complete lattice of sharp elements}

The notions of a sharp element and a sharply dominating effect algebra are due to S. Gudder $[8,9]$. An element $w$ of an effect algebra $E$ is called sharp if $w \wedge w^{\prime}=0$, and $E$ is called sharply dominating if for every $x \in E$ there exists a smallest sharp element $w$ with the property $x \leq w$.

The well known fact is that in every lattice effect algebra $E$ the subset $S(E)=\{w \in E \mid$ $\left.w \wedge w^{\prime}=0\right\}$ is a sub-lattice effect algebra of $E$ being an orthomodular lattice. Moreover if for $D \subseteq S(E)$ the element $\bigvee_{E} D$ exists then $\bigvee_{E} D \in S(E)$ hence $\bigvee_{S(E)} D=\bigvee_{E} D$. We say that $S(E)$ is a full sublattice of $E$ (see [11]).

Recall that elements $x, y$ of a lattice effect algebra $(E ; \oplus, 0,1)$ are called compatible (written $x \leftrightarrow y)$ iff $x \vee y=x \oplus(y \ominus(x \wedge y))$ (see [13]). $P \subseteq E$ is a set of pairwise compatible elements if $x \leftrightarrow y$ for all $x, y \in P$. $M \subseteq E$ is called a block of $E$ iff $M$ is a maximal subset of pairwise compatible elements. Every block of a lattice effect algebra $E$ is a sub-effect algebra and a sublattice of $E$ and $E$ is a union of its blocks (see [20]). Lattice effect algebra with a unique block is called an $M V$-effect algebra. Every block of a lattice effect algebra is an $M V$-effect algebra in its own right.

An element $z$ of an effect algebra $E$ is called central if $x=(x \wedge z) \vee\left(x \wedge z^{\prime}\right)$ for all $x \in E$. The center $C(E)$ of $E$ is the set of all central elements of $E$, see [7]. If $E$ is a lattice effect algebra then $z \in E$ is central iff $z \wedge z^{\prime}=0$ and $z \leftrightarrow x$ for all $x \in E$, see [17]. Thus in a lattice effect algebra $E, C(E)=B(E) \cap S(E)$, where $B(E)=\bigcap\{M \subseteq E \mid M$ is a block of $E\}$ is called a compatibility center of $E$. Evidently, $B(E)=\{x \in E \mid x \leftrightarrow y$ for all $y \in E\}$ and $B(E)$ is an $M V$-effect algebra. Hence $C(E)$ is a Boolean algebra, see [7]. Moreover, $B(E), C(E)$ and every block $M$ are also full sub-lattices of a lattice effect algebra $E$. Further, $B(E)$ and $C(E)$ are sub-effect algebras of $E$, see [7, 11, 21].

We are going to show that if the set $S(E)$ of an Archimedean atomic lattice effect algebra is a complete lattice then $E$ is isomorphic to a subdirect product of irreducible lattice effect algebras.

Definition 3. A direct product $\prod\left\{E_{\kappa} \mid \kappa \in H\right\}$ of effect algebras $E_{\kappa}$ is a cartesian product with $\oplus, 0,1$ defined "coordinatewise", i.e., $\left(a_{\kappa}\right)_{\kappa \in H} \oplus\left(b_{\kappa}\right)_{\kappa \in H}$ exists iff $a_{\kappa} \oplus_{\kappa} b_{\kappa}$ is defined for each $\kappa \in H$ and then $\left(a_{\kappa}\right)_{\kappa \in H} \oplus\left(b_{\kappa}\right)_{\kappa \in H}=\left(a_{\kappa} \oplus_{\kappa} b_{\kappa}\right)_{\kappa \in H}$. Moreover, $0=\left(0_{\kappa}\right)_{\kappa \in H}, 1=\left(1_{\kappa}\right)_{\kappa \in H}$.

A subdirect product of a family $\left\{E_{\kappa} \mid \kappa \in H\right\}$ of lattice effect algebras is a sublattice-effect algebra $Q$ (i.e., $Q$ is simultaneously a sub-effect algebra and a sublattice) of the direct product $\prod\left\{E_{\kappa} \mid \kappa \in H\right\}$ such that each restriction of the natural projection $\operatorname{pr}_{\kappa_{i}}$ to $Q$ is onto $E_{\kappa_{i}}$.

For every central element $z$ of a lattice effect algebra $E$, the interval $[0, z]$ with the $\oplus$ operation inherited from $E$ and the new unity $z$ is a lattice effect algebra in its own right. We are going to prove a statement about decompositions of $E$ into subdirect products of such intervals $[0, z]$ with $C([0, z])=\{0, z\}$ in the case when $E$ is an Archimedean atomic lattice effect algebra with $S(E)$ being a complete orthomodular lattice.

It was proved in [16, Lemma 2.7] that in every Archimedean atomic lattice effect algebra $E$ the subset $S(E)$ is a bifull sub-lattice of $E$, meaning that for any $D \subseteq S(E)$ the element $\bigvee_{S(E)} D$ exists iff the element $\bigvee_{E} D$ exists and they are equal. However, as M. Kalina proved, the center $C(E)$ of an Archimedean atomic lattice effect algebra $E$ need not be a bifull sub-lattice of $E$. Namely, if $C(E)$ is an atomic Boolean algebra, then $\bigvee_{E}\{p \in C(E) \mid p$ atom of $C(E)\}$ need not exist.

Definition 4. Let $\left(E ; \oplus_{E}, 0_{E}, 1_{E}\right)$ and $\left(F ; \oplus_{F}, 0_{F}, 1_{F}\right)$ be effect algebras. A bijective map $\varphi: E \rightarrow F$ is called an isomorphism if 
(i) $\varphi\left(1_{E}\right)=1_{F}$,

(ii) for all $a, b \in E: a \leq_{E} b^{\prime}$ iff $\varphi(a) \leq_{F}(\varphi(b))^{\prime}$ in such case $\varphi\left(a \oplus_{E} b\right)=\varphi(a) \oplus_{F} \varphi(b)$.

We write $E \cong F$. Sometimes we identify $E$ with $F=\varphi(E)$. If $\varphi: E \rightarrow F$ is an injection with properties (i) and (ii) then $\varphi$ is called an embedding.

Lemma 1. Let $E$ be an Archimedean atomic lattice effect algebra. The following conditions are equivalent:

(i) $C(E)$ is atomic and $\bigvee_{E}\{p \in C(E) \mid p$ atom of $C(E)\}=1$.

(ii) For every atom a of $E$ there exists an atom $p_{a}$ of $C(E)$ with $a \leq p_{a}$.

(iii) $E$ is isomorphic to a subdirect product of the family $\{[0, p] \mid p$ atom of $C(E)\}$.

Proof. $(i) \Longrightarrow($ iii): By [25, Theorem 3.1] the condition $(i)$ implies that $E$ is isomorphic to a subdirect product of the family $\{[0, p] \mid p$ atom of $C(E)\}$, since evidently $p_{1} \wedge p_{2}=0$ for every pair of mutually different atoms $p_{1}, p_{2}$ of $C(E)$.

$($ iii $) \Longrightarrow($ ii $)$ : By definition of the direct and subdirect products, if $a$ is an atom of $E$ then $a \in[0, p]$ for some atom $p$ of $C(E)$.

$($ ii $) \Longrightarrow(i)$ : Every Archimedean atomic lattice effect algebra $E$ is a union of its atomic blocks [15]. Let $M$ be an atomic block of $E$ and let $A_{M}=\{a \in M \mid a$ atom of $M\}$. Then every $a \in A_{M}$ is an atom of $E$. Otherwise, for $b \in E$ with $b<a$ we have $b<a \leq x$ or $b<a \leq x^{\prime}$ for all $x \in M$, as $a \leftrightarrow x$. It follows that $b \leftrightarrow x$. The maximality of blocks gives that $b \in M$, a contradiction.

Let $a \in A_{M}$. As $C(E) \subseteq S(E)$, if $a \leq z \in S(E)$ then $n_{a} a \leq z$. It follows that $a \leq p_{a} \Longrightarrow$ $n_{a} a \leq p_{a}$ since $p_{a} \in C(E) \subseteq S(E)$. Since by [16] $\bigvee_{E}\left\{n_{a} a \in M \mid a\right.$ atom of $\left.M\right\}=1$, we obtain that $\bigvee_{E}\{p \in C(E) \mid a$ atom of $C(E)\}=1$.

Theorem 1. Let $E$ be an Archimedean atomic lattice effect algebra. Let the set $S(E)$ of sharp elements of $E$ be a complete lattice. Then

(i) $C(E)$ is a complete Boolean algebra and a bifull sub-lattice of $E$.

(ii) $C(E)$ is atomic with $\bigvee_{E}\{p \in C(E) \mid p$ atom of $C(E)\}=1$.

(iii) For every atom a of $E$ there exists an atom $p$ of $C(E)$ with $a \leq p$.

(iv) $E$ is sharply dominating and $E$ is isomorphic to a subdirect product of irreducible lattice effect algebras.

Proof. $(i)$ : By [21], $S(E)$ is a full sublattice of $E$. By [16, Lemma 2.7], in every Archimedean atomic lattice effect algebra $E$ the set $S(E)$ is a bifull sub-lattice of $E$. Let $D \subseteq C(E)$. Since $S(E)$ is complete and $C(E)=S(E) \cap B(E) \subseteq S(E)$ there exists $\bigvee_{S(E)} D=\bigvee_{E} D \in C(E)$. This proves that $\bigvee_{C(E)} D=\bigvee_{E} D$, which proves that $C(E)$ is a complete and bifull sub-lattice of $E$.

(ii), (iii): Let $a$ be an atom of $E$ and let $p_{a}=\bigwedge_{C(E)}\{z \in C(E) \mid a \leq z\}=\bigwedge_{E}\{z \in C(E) \mid$ $a \leq z\}$, using $(i)$. Assume that there exists $c \in C(E), c \neq 0$ and $c<p_{a}$. Then $c \in B(E)$ and hence $c \leftrightarrow a$, which gives that $a \leq c$ or $a \leq c^{\prime}$. Since $a \leq c \Longrightarrow p_{a} \leq c<p_{a}$ and $a \leq c^{\prime}$ $\Longrightarrow c<p_{a} \leq c^{\prime}$, a contradiction in both cases. Hence $c \in C(E)$ with $0 \neq c<p_{a}$ does not exist. It follows that $p_{a}$ is an atom of $C(E)$ such that $a \leq p_{a}$. By (i), $1=\bigvee_{C(E)}\{p \in C(E) \mid$ $p$ atom of $C(E)\}=\bigvee_{E}\{p \in C(E) \mid p$ atom of $C(E)\}$.

$(i v)$ : Since $S(E)$ is a complete and bifull sub-lattice of $E$, the lattice effect algebra $E$ is sharply dominating. Moreover, $E$ is isomorphic to a subdirect product of lattice effect algebras by Lemma 1. Let us show that, for every atom $p$ of $C(E)$, the interval $[0, p]$ is an irreducible lattice effect algebra. 
Assume to the contrary that there exist $p_{0} \in A_{C(E)}=\{p \in C(E) \mid p$ atom of $C(E)\}$ and $x_{p_{0}} \in E$ such that $x_{p_{0}} \in C\left(\left[0, p_{0}\right]\right)$ and $0<x_{p_{0}}<p_{0}$. Let $x=\left(x_{p}\right)_{p \in A_{C(E)}}$ be such that $x_{p}=0$ for all $p \neq p_{0}$. Then $x \wedge x^{\prime}=0$. Further, let $y \in E$ be an arbitrary. Then $y=\left(y_{p}\right)_{p \in A_{C(E)}}=\bigvee_{E}\left\{y_{p} \mid p \in A_{C(E)}\right\}$, which gives that $x \leftrightarrow y$ as $x_{p} \leftrightarrow y_{p}$ for every $p \in A_{C(E)}$ (see [11]). Thus $x \in S(E) \cap B(E)=C(E)$ which contradicts $0<x_{p_{0}}<p_{0}$.

Note that in every complete (hence in every finite) lattice effect algebra $E$ the set $S(E)$ is a complete sub-lattice of $E$ (see [11]). Let us show an example of an Archimedean atomic $M V$-effect algebra $M$ which $S(M)$ is not a complete lattice.

Example 1. Simplest examples of $M V$-effect algebras are finite chains $M_{k}=\left\{0_{k}, a_{k}, 2 a_{k}, \ldots\right.$, $\left.n_{a_{k}} a_{k}=1_{k}\right\}, k=1,2, \ldots$ In this case $S\left(M_{k}\right)=C\left(M_{k}\right)=\left\{0_{k}, 1_{k}\right\}$ and $B\left(M_{k}\right)=M_{k}$, for $k=1,2, \ldots$ Let $M$ be a direct product of a family $\left\{M_{k} \mid k=1,2, \ldots\right\}$ of $M V$-effect algebras. Then $\oplus, 0,1$ on $M=\prod_{k=1}^{\infty} M_{k}$ are defined "coordinatewise" (see Definition 3).

It follows that if $x=\left(x_{k}\right)_{k=1}^{\infty} \in M$, hence $x_{k} \in M_{k}(k=1,2, \ldots)$ then $x^{\prime}=\left(x_{k}^{\prime}\right)_{k=1}^{\infty}$. If $x_{k} \neq 0_{k}$ for at most finite set of $k \in\{1,2, \ldots\}$ then $x$ is called a finite element. Clearly $x^{\prime}$ is finite iff $x_{k} \neq n_{a_{k}} a_{k}$ for at most finite set of $k \in\{1,2, \ldots\}$.

Let $M^{*}=\left\{x \in M \mid\right.$ either $x$ or $x^{\prime}$ is finite $\}$. We can easily see that $M^{*}$ is a sub- $M V$ effect algebra (i.e., a sub-lattice and a sub-effect algebra of $E$ ). Evidently $M^{*}$ as well as $S\left(M^{*}\right)=C\left(M^{*}\right)$ are not complete. Nevertheless, $\bigvee_{M}\left\{p \in C\left(M^{*}\right) \mid p\right.$ atom of $\left.C\left(M^{*}\right)\right\}=1$. Here $p \in C\left(M^{*}\right)$ is an atom of $C\left(M^{*}\right)$ iff $p=\left(p_{k}\right)_{k=1}^{\infty}$ such that there exists $k_{0}$ with $p_{k_{0}}=n_{a_{k_{0}}} a_{k_{0}}$ and $p_{k}=0$ for all $k \neq k_{0}$.

Recently, M. Kalina showed an example of an Archimedean atomic lattice effect algebra $E$ with atomic $C(E)$ for which the element $\bigvee_{E}\{p \in C(E) \mid p$ atom of $C(E)\}$ does not exist (see [12]). Thus, by Theorem $1(i i), S(E)$ is not a complete lattice.

\section{Applications in questions about the existence of faithful states on modular lattice effect algebras}

If a lattice effect algebra $E$ is a modular lattice then $E$ is called a modular lattice effect algebra. Recall the definition of a state on effect algebras.

Definition 5. A map $\omega: E \rightarrow[0,1]$ is called a state on the effect algebra $E$ if $(i) \omega(1)=1$ and (ii) $\omega(x \oplus y)=\omega(x)+\omega(y)$ whenever $x \oplus y$ exists in $E$. If, moreover, $E$ is lattice ordered then $\omega$ is called subadditive if $\omega(x \vee y) \leq \omega(x)+\omega(y)$, for all $x, y \in E$.

Note that a state on a lattice effect algebra need not be subadditive, while every state on an $M V$-effect algebra is subadditive. In [23] it was proved that a state $\omega$ on a lattice effect algebra $E$ is subadditive iff $\omega$ is a valuation, meaning that, for any $x, y \in E, x \wedge y=0$ implies $\omega(x \vee y)=\omega(x)+\omega(y)$.

A state $\omega$ on an effect algebra $E$ is called faithful if $\omega(x)>0 \Longrightarrow x>0$ for any $x \in E$.

An Archimedean effect algebra is called separable if every $\oplus$-orthogonal system $M$ of elements of $E$ (meaning that for every finite subset $\left\{x_{1}, x_{2}, \ldots, x_{n}\right\} \subseteq M$ there exists $x_{1} \oplus x_{2} \oplus \cdots \oplus x_{n}$ ) is at most countable.

Theorem 2. Let $E$ be a separable Archimedean atomic modular lattice effect algebra. Let the set $S(E)$ of sharp elements of $E$ be a complete lattice. Then there exists a faithful state on $E$.

Proof. By Theorem 1, $C(E)$ is a complete atomic Boolean algebra. Clearly, the orthomodular lattice $S(E)$ and the Boolean algebra $C(E)$ are separable. Hence $C(E)$ has only countably many 
atoms. It follows that there exists an (o)-continuous faithful state $\omega$ on $C(E)$. Since $C(E)$ is a Boolean algebra, the state $\omega$ is subadditive, which is equivalent to the fact that $\omega$ is a valuation (see [23, Theorem 2.3]). This implies the existence of an (o)-continuous faithful state on $S(E)$ (see [27, Theorem 5, p. 91]). Since $S(E)$ is a complete lattice, which is a bifull sub-lattice of $E$ (see [16]), the effect algebra $E$ is evidently sharply dominating. Now, using Theorem 4.3 of [26] and its proof we obtain that there exists a faithful state on $E$, as $n_{a} a \in S(E)$ for every atom $a$ of $E$.

\section{Atomic lattice effect algebras densely embeddable into complete lattice effect algebras}

It is well known that any partially ordered set $L$ can be embedded into a complete lattice $\widehat{L}$ by an algebraic method called MacNeille completion (or completion by cuts). It was proved in [28] that any complete lattice $K$ into which $L$ can be join-densely and meet-densely embedded is isomorphic to its MacNeille completion $\widehat{L}$. It means that to every element $x \in L$ there exist $M, Q \subseteq L$ such that $x=\bigvee_{\widehat{L}} \varphi(M)=\bigwedge_{\widehat{L}} \varphi(Q)$ (here we usually identify $L$ with $\varphi(L)$, where $\varphi: L \rightarrow \widehat{L}$ is the embedding).

However, there are lattice effect algebras $(E ; \oplus, 0,1)$ for which a complete lattice effect algebra $(\widehat{E} ; \widehat{\oplus}, 0,1)$ with above mentioned properties does not exist $(\oplus$ from $E$ cannot be extended onto $\widehat{E}$, see [18]).

In this part we study properties of central atoms of atomic lattice effect algebras for which the MacNeille completion $(\widehat{E} ; \widehat{\oplus}, 0,1)$ exists (see [18] for necessary and sufficient conditions for that). Note that if this is the case, then $E$ is Archimedean because every complete lattice effect algebra is Archimedean ([18, Theorem 3.3]).

Lemma 2. Let $(E ; \oplus, 0,1)$ be an atomic lattice effect algebra and let $(\widehat{E} ; \widehat{\oplus}, 0,1)$ be its MacNeille completion. Then

(i) $E$ and $\widehat{E}$ are Archimedean atomic lattice effect algebra with the same set of atoms $A_{E}$.

(ii) To every maximal subset of pairwise compatible atoms $A \subseteq A_{E}=A_{\widehat{E}}$ uniquely exist an atomic block $M$ of $E$ and an atomic block $\widehat{M}$ of $\widehat{E}$ such that $\widehat{M} \cap E=M$ and $\widehat{M}$ is the MacNeille completion of the block $M$.

(iii) $C(\widehat{E})$ is an atomic Boolean algebra.

(iv) $S(\widehat{E})$ is the MacNeille completion of $S(E)$ and $S(E)=S(\widehat{E}) \cap E$.

$(v) S(E)$ is atomic iff $S(\widehat{E})$ is atomic and then their sets of all atoms coincide.

Proof. We identify $E$ with $\varphi(E) \subseteq \widehat{E}$, where $\varphi: E \rightarrow \widehat{E}$ is the embedding (see [28]).

(i): Since $E \subseteq \widehat{E}$, we have that $A_{E} \subseteq A_{\widehat{E}}$, where $A_{E}$ and $A_{\widehat{E}}$ are sets of atoms of $E$ and $\widehat{E}$, respectively. Further the fact that $E$ is join-dense in $\widehat{E}$ implies that $x=\bigvee_{\widehat{E}}\{y \in E \mid y \leq x\}$, for every $x \in \widehat{E}$, which gives that $A_{\widehat{E}} \subseteq E$ and hence $A_{E}=A_{\widehat{E}}$. By [19, Theorem 3.3] $\widehat{E}$ is Archimedean, which implies that $E$ is also Archimedean.

(ii): By [15] every Archimedean atomic lattice effect algebra $E$ is a union of its atomic blocks $M$ which uniquely correspond to a maximal subset $A_{M}$ of pairwise compatible atoms of $E$. Thus $A_{M} \subseteq M \subseteq \widehat{M}$ and since $\widehat{M}$ is a complete $M V$-effect algebra with the same set of atoms as $M$ we obtain that $\widehat{M}$ is the MacNeille completion of the block $M$ by the Schmidt characterization (see [28]).

(iii): Since every complete atomic lattice effect algebra is orthocomplete, it has atomic center (see $[10])$, we obtain that $C(\widehat{E})$ is atomic. 
(iv): Let $y \in S(\widehat{E})$. Then there exists a set $\left\{a_{\kappa} \mid \kappa \in H\right\} \subseteq A_{\widehat{E}}=A_{E}$ such that $y=$ $\bigoplus_{\widehat{E}}\left\{a_{\kappa} \mid \kappa \in H\right\}=\bigvee_{\widehat{E}}\left\{a_{\kappa} \mid \kappa \in H\right\}$ (see [24, Theorem 3.3]). Since $\left\{a_{\kappa} \mid \kappa \in H\right\} \subseteq A_{E} \subseteq E$ we obtain that $\left\{n_{a_{\kappa}} a_{\kappa} \mid \kappa \in H\right\} \subseteq S(E)$, hence $S(E)$ is join-dense in $S(\widehat{E})$ which gives that $S(\widehat{E})$ is the MacNeille completion of $S(E)$ by [28]. Further $S(\widehat{E}) \cap E \subseteq S(E)$, thus $S(\widehat{E}) \cap E \subseteq S(E) \subseteq S(\widehat{E}) \cap E$.

$(v)$ : This follows by $(i v)$ and the Schmidt characterization of the MacNeille completion (see [28]) using the same arguments as in the proof of $(i)$.

Example 2. It is well known that there are even finite orthomodular lattices admitting no states (see [6]). On the other hand, every orthomodular lattice $(L ; \vee, \wedge, \perp, 0,1)$ or a Boolean algebra can be organized into a lattice effect algebra if we define a partial binary operation $\oplus$ on $L$ by: $x \oplus y=x \vee y$ for every pair $x, y \in L$ such that $x \leq y^{\perp}$. This is the original idea of G. Boole, who supposed that $x+y$ denotes the logical disjunction of $x$ and $y$ when the logical conjunction $x y=0$, (see [1]). For this lattice effect algebra $(L ; \oplus, 0,1)$ the compatibility center $B(L)=\bigcap\{B \subseteq L \mid B$ block of $L\}$ is evidently a Bolean algebra. In spite of that a state on $L$ need not exist.

Example 3. In general, for a lattice effect algebra $E$, the compatibility center $B(E)=\bigcap\{B \subseteq$ $E \mid B$ block of $E\}$ is an $M V$-effect algebra, as blocks of $E$ are $M V$-effect algebras, (see [20]). Hence $B(E)$ is not a Boolean algebra iff $B(E) \nsubseteq S(E)$. For instance, assume that $E_{1}$ is a horizontal sum of chains $\left\{0_{1}, a_{1}, 2 a_{1}=1_{1}\right\}$ and $\left\{0_{2}, a_{2}, 2 a_{2}=1_{2}\right\}$ and we identify zero and top elements. Let $E=\{0, c, 2 c\} \times E_{1}$ be a direct product of a chain $\{0, c, 2 c\}$ and the lattice effect algebra $E_{1}$. Then $E$ is a lattice effect algebra with two blocks $M_{1}=\{0, c, 2 c\} \times\left\{0_{1}, a_{1}, 2 a_{1}\right\}$ and $M_{2}=\{0, c, 2 c\} \times\left\{0_{2}, a_{2}, 2 a_{2}\right\}$. Hence $B(E)=M_{1} \cap M_{2} \simeq\{0, c, 2 c\}$ is not a Boolean algebra.

Theorem 3. Let $E$ be an atomic lattice effect algebra densely embeddable into a complete lattice effect algebra $\widehat{E}$. If $B(E)$ is not a Boolean algebra then there exists an (o)-continuous subadditive state on $E$.

Proof. Since every complete lattice effect algebra is Archimedean (see [19, Theorem 3.4]) so $\widehat{E}$ is Archimedean and the effect algebra $E$ is Archimedean, as $E \subseteq \widehat{E}$. By [15] $B(E)=$ $\bigcap\{M \subseteq E \mid M$ atomic block of $E\} \subseteq \bigcap\{\widehat{M} \subseteq \widehat{E} \mid \widehat{M}, M$ atomic block of $E\}=B(\widehat{E})$, by Lemma 2 (ii). Clearly, $B(E)$ is not a Boolean algebra iff there exists $x \in B(E)$ such that $x \wedge x^{\prime} \neq 0$, which gives $x \in B(\widehat{E})$ and $x \notin S(\widehat{E})$. Further, $C(\widehat{E})$ is atomic (Lemma $2(i i i)$ ) and $\bigvee_{\widehat{E}}\{p \in C(\widehat{E}) \mid p$ atom of $C(\widehat{E})\}=1$, which implies that $\widehat{E} \cong \prod\{[0, p] \mid p$ atom of $C(\widehat{E})\}$ and $B(\widehat{E}) \cong \prod\{B([0, p]) \mid p$ atom of $C(\widehat{E})\}$. By [16, Theorem 4.1], $B([0, p])=\{0, p\}$ or $[0, p]=\left\{0, a, 2 a, \ldots, n_{a} a\right\}$ for some atom $a \in E$. Thus, there exists an atom $p_{0}$ of $C(\widehat{E})$ such that $B\left(\left[0, p_{0}\right]\right) \neq\left\{0, p_{0}\right\}$ and hence $\left[0, p_{0}\right]=\left\{0, a, 2 a, \ldots, n_{a} a\right\}$ for some atom $a \in E$. It follows (see also [16, Theorem 5.8]) that there exists an (o)-continuous subadditive state $\widehat{\omega}$ on $\widehat{E}$ and hence $\omega=\widehat{\omega} / E$ is an $(o)$-continuous subadditive state on $E$ such that $\omega(a)=\frac{1}{n_{a}}$.

Note that conditions of Theorem 3 safeguard that an (o)-continuous subadditive state $\omega_{a}$ on $E$ exists for every atom $a \in E$ such that $a \in B(E)$ and moreover, that at least one such atom $a$ of $E$ exists, as $B(E) \neq C(E)$.

Corollary 1. Let $E$ be an Archimedean atomic lattice effect algebra with finitely many blocks. If $B(E)$ is not a Boolean algebra (equivalently there exists $x \in B(E)$ such that $x \wedge x^{\prime} \neq 0$ ) then there exists an (o)-continuous subadditive state on $E$.

Proof. It follows from the fact that a lattice effect algebra with finitely many blocks is densely embeddable into a complete lattice effect algebra iff $E$ is Archimedean (see [19, Theorem 4.5]). 


\section{Acknowledgements}

This work was supported by the Slovak Research and Development Agency under the contract No. APVV-0375-06 and the grant VEGA-2/0032/09 of MS SR. The author wishes to express his thanks to referees for many stimulating questions and suggestions during the preparation of the paper, which helped to improve it.

\section{References}

[1] Boole G., An investigation of the laws of thought, Macmillan, Cambridge, 1854 (reprinted by Dover Press, New York, 1967).

[2] Busch P., Lahti P.J., Mittelstaedt P., The quantum theory of measurement, Lecture Notes in Physics, New Series m: Monographs, Vol. 2, Springer-Verlag, Berlin, 1991.

[3] Dvurečenskij A., Pulmannová S., New trends in quantum structures, Mathematics and its Applications, Vol. 516, Kluwer Academic Publishers, Dordrecht; Ister Science, Bratislava, 2000.

[4] Foulis D.J., Bennett M.K., Effect algebras and unsharp quantum logics, Found. Phys. 24 (1994), 1331-1352.

[5] Foulis D.J., Effects, observables, states, and symmetries in physics, Found. Phys. 37 (2007), 1421-1446.

[6] Greechie R.J., Orthomodular lattices admitting no states, J. Combinatorial Theory Ser. A 10 (1971), 119132.

[7] Greechie R.J., Foulis D.J., Pulmannová S., The center of an effect algebra, Order 12 (1995), 91-106.

[8] Gudder S.P., Sharply dominating effect algebras, Tatra Mt. Math. Publ. 15 (1998), 23-30.

[9] Gudder S.P., S-dominating effect algebras, Internat. J. Theoret. Phys. 37 (1998), 915-923.

[10] Jenča G., Pulmannová S., Orthocomplete effect algebras, Proc. Amer. Math. Soc. 131 (2003), $2663-2671$.

[11] Jenča G., Riečanová Z., On sharp elements in lattice ordered effect algebras, BUSEFAL 80 (1999), 24-29.

[12] Kalina M., On central atoms of Archimedean atomic lattice effect algebras, submitted.

[13] Kôpka F., Chovanec F., Boolean D-posets, Internat. J. Theoret. Phys. 34 (1995), 1297-1302.

[14] Ludwig G., Die Grundlagen der Quantenmechanik, Springer-Verlag, Berlin, 1954 (translated by C.A. Hein, Springer-Verlag, Berlin, 1983).

[15] Mosná K., Atomic lattice effect algebras and their sub-lattice effect algebras, J. Electrical Engineering 58 (2007), 7/S, 3-6.

[16] Paseka J., Riečanová Z., The inheritance of BDE-property in sharply dominating lattice effect algebras and (o)-continuous states, Soft Comput., to appear.

[17] Riečanová Z., Subalgebras, intervals and central elements of generalized effect algebras, Internat. J. Theoret. Phys. 38 (1999), 3209-3220.

[18] Riečanová Z., MacNeille completions of D-posets and effect algebras, Internat. J. Theoret. Phys. 39 (2000), 859-869.

[19] Riečanová Z., Archimedean and block-finite lattice effect algebras, Demonstratio Math. 33 (2000), 443-452.

[20] Riečanová Z., Generalization of blocks for D-lattices and lattice-ordered effect algebras, Internat. J. Theoret. Phys. 39 (2000), 231-237.

[21] Riečanová Z., Orthogonal sets in effect algebras, Demonstratio Math. 34 (2001), 525-532.

[22] Riečanová Z., Proper effect algebras admitting no states, Internat. J. Theoret. Phys. 40 (2001), 1683-1691.

[23] Riečanová Z., Lattice effect algebras with (o)-continuous faithful valuations, Fuzzy Sets and Systems $\mathbf{1 2 4}$ (2001), no. 3, 321-327.

[24] Riečanová Z., Smearings of states defined on sharp elements onto effect algebras, Internat. J. Theoret. Phys. 41 (2002), 1511-1524.

[25] Riečanová Z., Subdirect decompositions of lattice effect algebras, Internat. J. Theoret. Phys. 42 (2003), 1415-1433.

[26] Riečanová Z., Wu J., States on sharply dominating effect algebras, Sci. China Ser. A 51 (2008), 907-914.

[27] Sarymsakov T.A., Ayupov Sh.A., Khadzhiev Dzh., Chilin V.I., Ordered algebras, FAN, Tashkent, 1983 (in Russian).

[28] Schmidt J., Zur Kennzeichnung der Dedekind-MacNeilleschen Hülle einer geordneten Hülle, Arch. Math. (Basel) 7 (1956), 241-249. 